\title{
STRUCTURE, CHEMISTRY AND PROPERTIES OF MINERAL NANOPARTICLES
}

\author{
Glenn Waychunas ${ }^{1}$, Hengzong Zhang ${ }^{2}$ and Ben Gilbert ${ }^{1}$ \\ ${ }^{1}$ Lawrence Berkeley National Laboratory \\ ${ }^{2}$ University of California, Berkeley
}

Nanoparticle properties can depart markedly from their bulk analog materials, including large differences in chemical reactivity, molecular and electronic structure, and mechanical behavior. The greatest changes are expected at the smallest sizes, e.g. $10 \mathrm{~nm}$ and below, where surface effects are expected to dominate bonding, shape and energy considerations. The precise chemistry at nanoparticle interfaces can have a profound effect on structure, phase transformations, strain, and reactivity. Certain phases may exist only as nanoparticles, requiring transformations in chemistry, stoichiometry and structure with evolution to larger sizes. In general, mineralogical nanoparticles have been little studied.

\section{WHAT'S REALLY DIFFERENT ABOUT NANOPARTICLES}

Nanoparticles, particularly those with diameters of less than $10 \mathrm{~nm}$, have a high proportion of atoms near their surfaces (ca. 16\% for a $10 \mathrm{~nm}$ cube). Because of this, several important aspects of surfaces can drive deviations from bulk structure and chemistry at different size scales. Size also directly affects a number of other bulk properties due to restriction on wave function radius, separation of defects and interacting strain fields, the relative overall dominance of bulk or surface energy, and changes in vibrational properties. Studies of nanoparticles connect with several other important scientific areas dealing with lower 
dimensional materials, especially the study of mineral and other solid surfaces, and investigations of the nucleation and initial growth of precipitates. Indeed, application of classical nucleation theory (CNT) indicates that all substances must "pass through” a nanoparticle size regime, either at or just after nucleation. As nucleation is generally a crucial rate-controlling step in precipitation, comprehension of nanoparticle characteristics can be key to evaluating the kinetics of precipitation and other phase transformations.

Another unusual aspect of nanoparticles is the way in which they may aggregate, and this follows from the type of forces in play between nanoparticles in solution. Under certain conditions nanoparticles can assemble in a crystallographically oriented mode, leading to a type of crystal growth with different kinetics than classical "layer by layer” growth, and consequently differing growth forms and habits. Such assembly may have special significance for the incorporation of natural impurities (Waychunas et al. 2005).

\section{Size matters}

Many characteristics of nanoparticles ensue from their size alone. Using equilibrium thermodynamics, one can show that if the surface energies of polymorphs differ significantly, that at small enough sizes the order of phase stability can be changed (Banfield and Zhang 2001). This is well known for $\mathrm{TiO}_{2}$, where anatase is the stable phase only for small particle sizes (Banfield and Zhang 1998). Another effect of size is change in crystallite habit. Nanoparticles of ceria $\left(\mathrm{CeO}_{2}\right)$ below about $10 \mathrm{~nm}$ have a truncated octahedron habit with $\{100\}$ and $\{111\}$ faces, but at larger sizes shift to the pure octahedron $\{111\}$ (Wang and Feng 2003). The most stable forms calculated for zirconia nanoparticles have habit changes at 8 and $12.7 \mathrm{~nm}$ diameters (Barnard et al. 2006). 
The property of quantum (electron) confinement (QC), most notably observed in metal and narrow band gap semiconducting nanoparticles, is another size-related property. In this case the delocalized wavefunction associated with an excitonic state (i.e. a band gap transition to the lowest states in the conduction band) may be restricted in extent at small diameters, raising its energy relative to the bulk (Kayanuma 1988). Perhaps the clearest example is in materials such as CdSe, where a noticeable color change is observed. The effect can occur in any material, but is subject to limitations. Purely insulating materials having a large band gap with low quantities of impurities will have localized dopant wavefunctions which may be too far apart to overlap. Hence the properties of the dopant electronic states will not be subject to QC. Excitonic transitions in such materials will be higher in energy than UV reaching earth' surface, and hence unimportant for natural processes. Hence corundum, quartz, carbonates and many other mineral nanoparticles in the pristine state would not be expected to show any effect unless doped in particular ways. The smaller band gap of hematite (ca. $2.1 \mathrm{eV}$ ) has been shown to increase in nanoparticles to about $2.5 \mathrm{eV}$ (Vayssieres et al. 2005). The band gap of maghemite also increases in nanoparticles, but in ferrihydrite nanoparticles there is no observed increase. These changes are likely due to surface state modifications and structural disorder rather than to QC effects, but the true origins of these shifts is not yet certain. Small nanoparticles of mineral oxides are not, in general, "quantum dots" or "quantum wires" as sometimes suggested in the literature, as it cannot be generally assumed that a QC effect will be observed.

Both surface charge and surface bond relaxation can effect the electronic energy states of surface species. For small enough particles this effect can be large, and shifts in band gap or band edge position up or down are possible due to the shifting of states near the top of the valence band, or bottom of the conduction band. Surface relaxation or reorganization can also 
possibly generate new electronic states within the band gap. Indeed, sorption of species can possibly affect the electronic state energies so that reactivity may be changed both from functional group concentration, and modified electronic transfer properties. Some changes that may be due to these effects have been noted in nanogoethite, nanohematite and ferrihydrite particles, and spectral measurements show a shift of energy band positions as a function of particle size. Much new work is needed to understand these changes, as neither the precise structural variations with size, nor the effect of particular variations on electronic structure are known.

Surfaces also perturb coupling between magnetic ions, so that small particles of magnetic phases would be expected to have magnetic properties dependent on size. This is certainly true for nanoparticle goethite and hematite, where bulk antiferromagnetic coupling is reduced and superparamagnetic activity is enhanced with reduced size (Carbone et al. 2005). An excellent review of the subject by Rancourt (2001) is available.

Still another size effect is solubility, which in large part controls the stability of environmental nanoparticles. It follows from CNT that larger particles are more stable than smaller critical nuclei, and that the stabilizing energy increases with size. It can be shown from strictly kinetic analysis that this requires smaller particles to be more soluble than their larger brethren, and this is the basis for Ostwald ripening. The effect assumes surface energy independent of particle size (the capillarity assumption), given no other structure changes, which is a sound premise for larger particles. However this assumption is debatable for particles near critical nucleus size, and is certainly untrue if there are surface structure and chemical changes with size. Hence Ostwald ripening may not proceed classically in the nano regime. 


\section{STRUCTURAL CHARACTERIZATION OF NANOPARTICLES}

While many experimental properties can be obtained from an ensemble of aggregated nanoparticles, this reduces our ability to understand the precise effects of shape, size, distortion and bonding deficit due to both averaging and grain-grain interactions. A related problem is due to polydispersity, or the range of sizes within any one sample of dispersed nanoparticles.

Neglecting these problems, average structural parameters can be obtained from x-ray scattering and extended x-ray absorption fine structure (EXAFS) analysis, and electronic parameters from various types of x-ray spectroscopy. Over the last decade pair-distribution-analysis (PDF) has gained prominence because of gains produced by virtue of synchrotron source x-ray intensity. PDF analysis is essentially powder diffraction done to large wavevector Q (e.g. $40 \AA^{-1}$ or more), equivalent to using a very short x-ray wavelength with significant scattering angles. The narrow divergence of the x-ray beam along with the small energy spread leads to scattering patterns of unprecedented quality, and the Fourier transform of the weighted structure factor function can yield well-defined interatomic distances as large as 3 or $4 \mathrm{~nm}$ or more (Gateshki et al. 2007). This capability allows definition of particle size directly from the PDF (Figure 1), if one can allow for shape effects (Kodama et al. 2006). Another effect of a large $Q$ range is the $Q^{-1}$ dependence of peak widths in the distribution function, so that atomic shells are readily separated at the smaller interatomic distances, and better resolved at all distances. This allows detailed analysis of interatomic distance changes as a function of radius, composition, and so forth.

EXAFS analysis is complimentary, but contains less information, with an effective Q range of about $18 \AA^{-1}$, and a dramatic loss in signal quality with Q. However EXAFS analysis is 
pair specific (e.g. Fe-X) which can be advantageous in phases with many constituents or with a specific constituent at low concentrations. If the nanoparticles can be oriented, or have a high degree of texturing, polarized EXAFS spectra can be obtained, allowing directional correlation information to be extracted. This approach may be most useful in examination of the structure of nanoparticles forming on surfaces (Waychunas et al. 1999). EXAFS also has the advantage of being able to examine nanoparticles in solution, in melts, or under conditions unsuitable for many other structural techniques.

Electron diffraction in a transmission electron microscope (TEM) is another powerful approach, especially if single nanoparticles can be examined and the vacuum conditions and relative high energy dosage is unimportant (i.e. non-hydrous, relatively strongly bonded phases). Recent work by Huang et al. (2008) combining TEM studies with molecular dynamics simulations demonstrates that the surface binding deficit in gold nanoparticles creates surface relaxations (in this case contractions). These are observed for all surface species in decreasing magnitude for corner sites $>$ edge sites $>$ face sites. Use of a focused nanobeam could allow analogous studies of nanograin polycrystalline materials (Dryer and Kirkland 2007).

Future developments in diffraction-based structural analysis, e.g. by use of a nextgeneration coherent x-ray sources, may permit single nanoparticle structure analysis, using a single x-ray pulse at the femtosecond time scale. The fortuitous development of high speed and superbright x-ray sources makes this nanocrystallography possible. The Howells coherent diffraction limit indicates that the smallest structural domain that can be imaged without destruction of the sample by radiation is about $10 \mathrm{~nm}$ (Marchesini et al. 2003). This limit is defeated by use of crystals with enormous numbers of identical unit cells, but that will not help the case with varied nanoparticles, that cannot be crystallized into "super” crystals. However if a 
single pulse of $\mathrm{x}$-rays can produce a diffraction pattern prior to charge-induced explosion of the particle, then the Howells limit can be breached for single nanocrystals (Robinson 2008).

A separate important consideration is measurement of the size of nanoparticles. X-ray scattering peak broadening (XSPB), dynamic light scattering (DLS), and small angle x-ray scattering (SAXS) are all useful with provisos (Waychunas 2001). DLS can yield rapid results, but slightly larger diameters due to its sensitivity to solvent volume which may be carried along by a nanoparticle. XSPB diameters are related to coherent scattering domain (CSD) size and not

strictly diameter. SAXS measurements are likely to be most accurate, but require more complex data analysis. All of these techniques can be standardized by TEM imaging, assuming the phases are stable in vacuum.

\section{STRUCTURE AND CHEMISTRY DIVERGENCE FROM BULK PHASES}

\section{Surface Crystal Chemistry}

Surfaces of all materials represent a balance between the complete bonding of atoms found in the bulk, with a certain proportion of bonding deficit at the surface. The most obvious display of this is seen in vacuum interfaces, which maximize the deficit. Figure 2 shows the (0001) surface of corundum as determined for vacuum- and hydrous atmosphere-equilibrated single crystals. The vacuum surface has terminal $\mathrm{Al}$ ions that are pulled down close to the oxygens by coulombic forces, and have only three oxygen neighbors. In contrast the hydrous surface has complete $\mathrm{Al}(\mathrm{O}, \mathrm{OH})_{6}$ polyhedra at the surface, a different set of relaxations of atomic 
positions, and a partially ordered water overlayer (Eng et al. 2000). The relaxations of atom positions relative to the bulk create differences in acid-base reactivity, and the formation of the ordered water overlayer may have other consequences for sorption, e.g. stabilization of "outersphere" complexes or of less energetically favorable "inner-sphere” sorbate topology (Waychunas et al. 1995).

Somewhat larger changes are observed in the surfaces of the corresponding isostructural phase, hematite, and differ on different surface planes, and as a function of preparation (Tanwar et al., 2007). Here a notable loss in Fe population is observed in samples equilibrated at ambient conditions, so that the stoichiometry is oxygen excess with additional protons for charge balancing. On the hematite r-plane (1-102) the surface (taken as the slabs of atoms above the stoichiometric repeat unit) has composition $\mathrm{FeOOH}$. It is tempting to use this information to make predictions about the corresponding nanoparticles. For example, as the surface region is about $1 \mathrm{~nm}$ thick (defined by significant relaxations with respect to bulk atomic positions), a 2 nm nanoparticle whose surface chemistry behaves like that in larger hematite crystals, would have goethite stoichiometry. In effect, hematite is unstable at this size. The single crystal termination also appears to feature a HO-Fe coordination environment that is more reactive than HO- $\mathrm{Fe}_{2}$ which would make up a simple terminated surface (as in corundum). Sintered nanohematite studied via Fourier Transform Infrared and Raman spectroscopy appears to have maghemite-like (i.e. spinel) surface defects, those these are lessened in quantity when the particles are exposed to water (Chernyshova et al. 2007). These comparisons suggest that nanoparticle surface structure, and therefore many nanoparticle properties, are highly dependent on formation conditions. It seems likely that the common phases we normally consider may be replaced by an entirely different set at small diameters. 
Besides stoichiometry, atomic surface relaxations alone have consequences for the incorporation of impurities and for acid-base properties. An expanded surface interatomic distance should allow larger ions to be dissolved near the surface, and vice versa. Hence natural nanocrystals may have surfaces enriched in appropriate sized species present during their formation. Potentially this would give rise to solvi that change with crystallite size in the nanometer regime.

Changes in acid-base character ensue from bond valence dependence on metal-oxygen

distance. For example, for surface site $\mathrm{H}-\mathrm{O}-\mathrm{M}^{3+}{ }_{2}$ with the $\mathrm{M}$ six-coordinated by oxygens, the nominal bond valence at the oxygen is 2 (proton=1, each $\mathrm{M}=0.5$ ). Such a proton is tightly bound and unreactive, and would resist forming other H-bonds. However if the O-M distance is relaxed, bond valence-bond distance relationships indicate that the oxygen becomes unsatisfied, i.e. bond valence $<2$. An exterior proton would then be needed to make H-bonds to the oxygen to make up the difference, and the site is more basic. Alternatively, shortening of the O-M distances would allow the existing proton to make H-bonds to other surface species, and the oxygen site is more acidic.

\section{Edges, corners and variable points of zero charge}

Besides being enriched in surface sites and surface-induced properties, nanoparticles must have a large proportion of “edge” and "corner” sites with potential bonding deficits larger than flat surfaces. This may lead to instability at smaller sizes, so that well defined "faces", “edges” and “corners” do not develop until the relative number 
of such sites is below a critical maximum probably determined by kinetic factors. Anecdotal evidence seems to support this notion inasmuch as TEM analysis of Fe oxide and oxyhydroxide nanoparticles below about $4 \mathrm{~nm}$ in size generally shows poor face development, while larger particles are often sharply defined. Molecular dynamics and ab initio simulations of such particles are only now gaining sufficient sophistication to tackle these issues, particularly in the presence of aqueous solution. Accurate simulation of the surface water is a difficult task in itself, and most work to date uses overly simplified water interactions or even no water at all (vacuum).

The bonding deficits of corner and edge sites may have important consequences for both reactivity and acid-base character. An oxygen site near one of these features will have fewer metal ions coordinated to it on average, and hence require more protonation for stability. As the protons would be more difficult to remove than for plane surfaces, these oxygens give rise to more basic sites. If this is correct then we might see the effect in powdered samples versus single crystals with point of zero charge determinations. For corundum the single crystal (0001) surface has a point of zero charge (PZC) of about $\mathrm{pH}=6.3$ while a powder (or an amorphous surface) has a PZC in the range 8.5-9.0 (Zhang et al. 2008). This indicates the protons are harder to remove from the presumed larger numbers of edge and corner sites in the powders (and mixture of all kinds of sites in the amorphous sample), as we predict. Hence in the absence of other factors, size and shape will partially control pKa and proton transfer reactions.

\section{CHANGES IN MECHANICAL PROPERTIES}


Another size effect is due to defect distributions within nanoparticles and has a major effect on mechanical properties. Thermodynamic analysis suggests that dislocations and stacking faults should be unstable in nanoparticles with respect to diffusion to the surface and annihilation. However such defects may be retained by kinetic factors, e.g. association with larger scale shape features derived from the assembly of smaller nanoparticle precursors (Banfield and Zhang 2001; Penn and Banfield 1998), or be trapped at nanoparticle grain boundaries. An important consideration is that the strain interaction from any two dislocations within the same nanoparticle may be quite large, as the strain field from a dislocation can extend beyond the 100 nanometer range. Hence nanoparticles large enough to have two or more dislocations may have significant interactions of strain fields and result in a relative decrease in compressibility relative to bulk material. The effect is well characterized in polycrystalline phases where as grain size decreases into the nanometer range, stiffness increases due to increased trapping of dislocations at interfaces (the Hall-Petch effect). On the other hand, at small enough sizes a single dislocation may be the maximum energetically possible and the particles would be more compressible (the inverse Hall-Petch effect), though the precise reason for this observed behavior is highly debated (Louchet et al. 2006). This suggests a non-linear dependence of compressibility with size, and the possibility of engineering unique multiphase composite materials with tuneable stiffness (Sun, 2007). Stiffening can also occur as a result of surface distortions owing to the bonding dissatisfaction we have described. In this case the strain can have a complex radially symmetric profile with several distinct components, which has been quantitatively analyzed in ZnS nanoparticles (Gilbert et al. 2004). This surface strain is related to the type of surface ligand, with more weakly bonded ligands resulting in a situation like that of the vacuum interface, i.e. large bonding deficits and unusual coordinations. For ZnS, methanol 
ligands are weakly bonded and produce sufficient surface distortion to create strain throughout the entire volume of a $3 \mathrm{~nm}$ nanoparticle, resulting in altered vibrational properties and stiffness (Figure 3). Ligand exchange with a more strongly bonded species, e.g. water, results in relaxation of strain and transformations of nanoparticle structure (Zhang et al., 2003).

\section{PURELY NANOSIZE PHASES: FERRIHYDRITE AND CLASSICAL ARGUMENTS}

Our discussion above raises the issue of phases that might exist naturally only at nanometer sizes. The best mineralogical example of this is ferrihydrite, whose structure can be described by a periodically repeating unit cell, but which does not appear to occur in coherent scattering domains (CSDs) larger than about $6 \mathrm{~nm}$ (Michel et al. 2007). Small CSDs are usually

indicative of high defect densities that interrupt structural continuence sufficiently with respect to x-ray scattering. This leads to broadened $\mathrm{x}$-ray scattering peaks. Complicating the structural description further, ferrihydrite has a stoichiometry change with size of crystallite, and site population on two of the three Fe sites decreases with radius (Michel et al. 2007). These observations seem consistent with the type of observations for the hematite r-plane surface, and suggest an increased structural role of water at smaller dimensions. In accord with this, thermodynamic measurements indicate that surface energies of nanoparticles are reduced by hydration, and that oxyhydroxides have lower surface energies than oxides (Navrotsky et al. 2008). 
One issue here is whether the description of such a small crystallite is truly distinct from a finite molecular crystal, i.e. whether there is actually translational symmetry of a bona fide unit cell. A model for the formation of ferrihydrite could involve nucleation around specific cluster motifs in solution, which cannot build coherently beyond a particular range due to strain buildup. This would be analogous to thin layers of semiconductor materials sputtered heteroepitaxially onto similar materials having slightly different unit cells. In such case the strain builds up as the layer thickness increases, eventually driving dislocation formation at the interface and loss of coherence (Jain et al. 1997).

Another interesting concept derives from CNT. In the ideal Volmer-Weber formulation the surface and volume free energy sum has a maximum at a critical size, decreasing for any larger volume. This assumes a surface energy independent of radius, as well as a homogeneous phase. However what happens if neither assumption is true? If in the nanoparticle the net stoichiometry varies with radius, then the surface and bulk energy will define a different CNT free energy curve, with a different critical nucleus size. Hence kinetic effects can result in different nanoparticle compositions from different synthesis conditions. An interesting question is whether there exists a "smooth" to "faceted" transition in some types of nanoparticles as observations on iron oxyhydroxide mineral suggest? This is not the case with Au and most metallic nanoparticles, and would not be expected as the binding energy of Au atoms is quite large and would overcome any surface energy effects. But for mineral nanoparticles dominated by metal oxygen bonds, this may be much more likely, and would have consequences for aggregation behavior and growth kinetics. 


\section{NANOPARTICLE SURFACE WATER, AGGREGATION AND THE KINETICS OF ORIENTED AGGREGATION}

Water near surfaces is well known to be ordered to varying degrees depending on surface charge, type of surface atoms, and atomic spacing. As water generally attempts to have a tetrahedral bond network, this can be frustrated if strong hydrogen bonds are formed at a surface that force different bond angles and local density. In the case of nanoparticles the zone of ordered or frustrated water can constitute a volume significant compared to the solid volume, and this may create unusual aggregation, sorption or other chemical effects.

One possible consequence of local ordered water is the dissipation of surface charge so that particles that would normally repel one another, e.g. at $\mathrm{pH}$ values away from the ZPC, might be able to closely approach one another. This seems to be the case for nanoparticles that undergo oriented aggregation (OA). In OA, nanoparticles can assemble into larger single crystals bypassing the usual route of atom by atom addition and layer by layer growth. Much evidence has been collected to show that OA occurs in a variety of systems, but at a size regime where measurements are difficult. The attachment appears to occur in the size regime where planar facets appear (Figure 4). In aqueous classical growth theory an adatom attaches to a growth surface by displacing sorbed water at a kink site. In OA a large amount of surface water must be displaced, but a large number of new bonds are also formed due to the perfection of the added nanoparticle. The frustration level of the surface bound water may act to decrease this dewatering energy, i.e. a water entropy change.

Though the energy barrier may be larger, crystallization via an OA process may be more rapid than via the classical process, as where atomic diffusion limits classical growth rate, the 
limiting step for $\mathrm{OA}$ is particle diffusion. Hence where solubility is low so that the concentration of solute is restricted, OA may be the most feasible growth mechanism. Kinetic studies show a trade off in OA vs OR mechanisms in ZnS and PbS nanoparticle growth (Zhang et al. 2007).

OA may also be favored when well defined monodispersed nanoparticles are created by particular precursor chemical pathways. As shown in Figure 4, submicron sized goethite particles show a pattern of density variations suggesting growth by OA. Hence, is there a precursor process that tends to produces goethite nanoparticles of uniform size? The answer is not yet clear, but general observations suggest a well-defined size where goethite nanoparticles assume sharply defined crystal faces, providing just the sorting factor needed to source the OA process.

\section{SILICA, SILICATES AND MORE COMMON MINERALS}

The nanoparticles studied most intensely to date have been largely dictated by commercial importance, ease of synthesis, and scientific novelty, and many common mineral phases have been neglected, so we next mention a few of these.

Silica clusters exist in natural waters due to the relatively high solubility of quartz at near neutral $\mathrm{pH}$ conditions and the ease of nucleating amorphous silica. Critical nuclei appear to be about $3 \mathrm{~nm}$ in diameter and have variable water content. The structure is usually termed opal-A, and this transforms with dewatering and reorganizing successively into opal-CT, opal-C, moganite, cristobalite, and finally, quartz (Icopini et al 2005; Herdianita et al. 2000). This process underlines the importance of metastability in natural nanoparticle structures, as well as 
the stabilizing effect of surface or structurally bound water molecules. However the precise structural role of water in silica nanoclusters is not well determined.

This stabilizing effect of water also has been demonstrated by molecular dynamics simulations of calcite nanoparticles, where crystallites as small as 90 total atoms had structures very similar to the bulk if sorbed water was allowed to complete the surface coordination environments. In analogous vacuum simulations the particles were extremely disordered, with significant $\mathrm{CO}_{3}$ group rotations and translations, and with the smallest such particles being essentially amorphous (Cooke and Elliott 2007). Significantly, on those parts of the crystallites that showed the most disorder in vacuum (with disorder decreasing in the order corner $>$ edge $>$ face) the water molecule resonance times were the shortest in the hydrous simulations. Hence the structural stability is closely correlated to the time-averaged bonding contribution of the hydration layer.

A nanoscale structure seen in natural minerals but not well understood has been termed a “nanoroll”, and refers to the rolled up layered silicate structures found in serpentine materials (Jancar and Suvorov 2006). Analogous structures can be formed from nickel oxides and vanadium oxides by dehydration of the related hydroxide sheet structures under hydrothermal conditions, and $\mathrm{TiO}_{2}$ nanotubes have been well characterized (Gateshke et al. 2007), but the formation of the serpentine nanorolls is likely rather different. A suggested mechanism of formation is the intercalation of water between structural layers during deformation followed by strain release through a mechanism featuring layer separation followed by coiling (Chivilikhin et al. 2007). Though not a property of separated nanoparticles, this kind of process relates stress reduction and hydration reactions to each other on the nanoscale, whereas entirely different strain release mechanisms ostensibly occur at larger scales. Hydrous silicates have been studied in 
detail by molecular dynamics simulation approaches, though not generally with attention to size or shape effects.

\section{SUMMARY}

Mineral nanoparticles, especially of few nm diameter, can differ significantly from bulk analogs. Chemistry, stoichiometry, surface structure, strain, and degree of order may all be functions of size and formation conditions. Major differences in properties can result from these variations, encompassing electronic, chemical, magnetic, and mechanical behavior. Holistic study of these structure-chemistry-property relationships yield insights into the bases for predicting and tailoring properties in manufactured materials, and discovering key environmental nanoparticlebiological connections.

\section{ACKNOWLEDGEMENTS}

The authors gratefully acknowledge the guidance and support from Prof. Jillian Banfield for a significant portion of the work presented here, and for development of the Nanogeoscience center at Lawrence Berkeley National Laboratory. Reviews by Andrew Madden, Michael Hochella, David Vaughan, and an anonymous reviewer are much appreciated. Much of the nanoscience research of GAW, HZ and BG has been supported through the U.S. Department of Energy, Office of Basic Energy Sciences, within the Chemical Sciences, Geosciences and Biosciences Program, Contract No. DE-AC02-05CH11231. 


\section{References}

1. Banfield JF and Zhang H (2001) Nanoparticles in the environment. Reviews in Mineralogy and Geochemistry 44: 1-58

2. Banfield JF and Zhang H (1998) Thermodynamic analysis of phase stability in nanocrystalline titania. Journal of Materials Chemistry 8:2073-2076

3. Barnard AS, Yeredla RR and Xu H (2006) Modelling the effect of particle shape on the phase stability of $\mathrm{ZrO}_{2}$ nanoparticles. Nanotechnology 17: 3039-3047

4. Chernyshova IV, Hochella MF, Madden AS (2007) Size-dependent structural transformations of hematite nanoparticles. 1.phase transition. Physical. Chemistry Chemical Physics 9: 1736-1750

5. Chivilikhin SA, Popov IY, Blinova IV, Kirillova SA, Konovalov AS, Oblogin SI, Tishkin VO, Chernov IA, Gusarov VV (2007) Simulation of the formation of nanorolls. Glass Physics and Chemistry 33: 315-319

6. Cooke DJ, Elliott JA (2007) Atomistic simulations of calcite nanoparticles and their interaction with water. Journal of Chemical Physics 127: 104706

7. Carbone C, Di Benedetto F, Marescotti P, Sangregorio C, Sorace L, Lima N, Romanelle M, Lucchetti G, Cipriani C (2005) Natural Fe-oxide and -oxyhydroxide nanoparticles: an EPR and SQUID investigation. Mineralogy and Petrology 85:19-32

8. Dwyer C, Kirkland AI (2007) Electron nanodiffraction using sharply focused parallel probes. Applied Physics Letters 90: 151104

9. Eng PJ, Trainor TP, Brown GE, Waychunas GA, Newville M, Sutton SR, and Rivers ML (2000) Structure of the hydrated alpha- $\mathrm{Al}_{2} \mathrm{O}_{3}$ (0001) surface. Science 288: 1029-1033 
10. Gateshki M, Chen Q, Peng L-M Chupas P, Petkov V (2007) Structure of nanosized materials by high-energy X-ray diffraction. Zeitschrift für Kristallographie. 222:612-616

11. Gilbert B, Huang F, Zhang H, Waychunas GA, Banfield JF (2004) Nanoparticles: Strained and Stiff. Science 305:651-654

12. Herdianita NR, Browne PR, Rodgers KA, Campbell KA (2000) Mineralogical and morphological changes accompanying aging of siliceous sinter. Mineralium Deposita 35: 48-62

13. Huang WJ, Sun R, Tao J, Menard LD, Nuzzo G, Zuo JM (2008) Coordination-dependent surface atomic contraction in nanocrystals revealed by coherent diffraction. Nature Materials 7:308-313

14. Icopini GA, Brantley SL, Heaney PJ (2005) Kinetics of silica oligomerization and nanocolloid formation as a function of $\mathrm{pH}$ and ionic strength at $25^{\circ} \mathrm{C}$. Geochimica et Cosmochimica Acta 69:293-303

15. Jain SC, Harker AH, Cowley RA (1997) Misfit strain and misfit dislocations in lattice mismatched epitaxial layers and other systems. Philosophical Magazine A 75: 14611515

16. Jancar B, Suvorov D (2006) The influence of hydrothermal-reaction parameters on the formation of chrysotile nanotubes. Nanotechnology 17: 25-29

17. Kayanuma Y (1988) Quantum size effects of interacting electrons and holes in semiconductor microcrystals with spherical shape. Physical Review B 38: 797-805

18. Kodama K, Iikubo S, Taguchi T, Shamoto S (2006) Finite size effects of nanoparticles on the atomic pair distribution functions. Acta Crystallographica A 62:444-453 
19. Levchenko AA, Li G, Boerio-Goates J, Woodfield BF, Navrotsky A (2006) $\mathrm{TiO}_{2}$ Stability Landscape: Polymorphism, Surface Energy, and Bound Water Energetics. Chemistry of Materials 18: 6324-6332

20. Louchet F, Weiss J, Richeton T (2006) Hall-Petch Law revisited in terms of collective dislocation dynamics. Physical Review Letters 97:075504

21. Marchesini S, Chapman H, Hau-Riege S, London R, Szoke A, He H, Howells M, Padmore H, Rosen R, Spence J, Weierstall U (2003) Coherent X-ray diffractive imaging: applications and limitations. Optics Express 11:2344-2353

22. Michel FM, Ehm L, Antao SM, Lee PL, Chupas PJ, Liu G, Strongin DR, Schoonen MA, Phillips BL, Parise JB (2007) The Structure of Ferrihydrite, a Nanocrystalline Material. Science 316:1726-1729

23. Navrotsky A, Mazeina L, Majzlan J (2008) Size-Driven Structural and Thermodynamic Complexity in Iron Oxides. Science 319: 1635-1638

24. Penn L and Banfield JF (1998) Imperfect Oriented attachment: Dislocation generation in defect-free nanocrystals. Science 281:969-971

25. Rancourt DG (2001) Magnetism of Earth, Planetary, and Environmental Nanomaterials. Reviews in Mineralogy and Geochemistry 44: 217-292

26. Robinson I (2008) Giant molecules or tiny crystals? Nature Materials 7: 275-276

27. Sun CQ (2007) Size dependence of nanostructures: Impact of bond order deficiency. Progress in Solid State Chemistry 35:1-159

28. Tanwar KS, Lo CS, Eng PJ, Catalano JG, Walko DA, Brown GE, Waychunas GA, Chaka AM, Trainor TP (2007) Surface diffraction study of the hydrated hematite (1-102) surface. Surface Science 601:460-474 
29. Vayssieres L, Sathe C, Butorin SM, Shuh DK, Nordgren J, Guo J (2005) OneDimensional Quantum-Confinement Effect in a-Fe2O3 Ultrafine Nanorod Arrays. Advanced Materials 17:2320-2323

30. Wang ZL, Feng X (2003) Polyhedral Shapes of $\mathrm{CeO}_{2}$ Nanoparticles. Journal of Physical Chemistry B 107:13563-13566

31. Waychunas GA (2001) Structure, Aggregation and Characterization of Nanoparticles. Reviews in Mineralogy and Geochemistry 44: 105-166.

32. Waychunas GA, Davis JA, Fuller CC (1995) Geometry of sorbed arsenate on ferrihydrite and crystalline FeOOH: Re-evaluation of EXAFS results and topological factors in predicting sorbate geometry, and evidence for monodentate complexes. Geochimica et Cosmochimica Acta 59: 3655-3661

33. Waychunas GA, Davis JA, Reitmeyer R (1999) Grazing-incidence EXAFS study of $\mathrm{Fe}^{3+}$ sorption on single crystal quartz substrates. Journal of Synchrotron Radiation 6:615-617

34. Waychunas GA, Kim CS, Banfield JF (2005) Nanoparticulate Iron Oxide Minerals in Soils and Sediments: Unique Properties and Contaminant Scavenging Mechanisms. Journal of Nanoparticulate Research 7: 409-433.

35. Zhang H, Gilbert B, Huang F, Banfield JF (2003) Water-driven structure transformations in nanoparticles at room temperature. Nature 424:1025-1029

36. Zhang J, Wang Y, Zheng J, Huang F, Chen D, Lan Y, Ren G, Lin Z, Wang C (2007) Oriented attachment kinetics for ligand capped nanocrystals: Coarsening of Thiol-capped PbS nanoparticles. Journal of Physical Chemistry B 111:1449-1454 
37. Zhang L, Tian C, Waychunas G, Shen YR (2008) Structures and Charging of $\alpha$-Alumina (0001)/Water Interfaces Studied by Sum-Frequency Vibrational Spectroscopy. Journal of the American Chemical Society 130: 7686-7694

\section{FIGURE CAPTIONS}

Figure 1. Neutron scattering PDF of spherical $\mathrm{TiO}_{2}$ nanoparticles (circles) compared with bulk calculation (grey) and truncated particle size model (black). The size distribution is derived by fitting the PDF data to calculated PDFs for each whole nm diameter. From Kodama et al. (2006) with permission from IUC.

Figure 2. Comparison of the corundum (0001) vacuum equilibrated termination (bottom) versus the termination equilibrated with a hydrous atmosphere (top), both determined via surface x-ray diffraction. Oxygen atoms are red, aluminum atoms are in white. A layer of bonded water is observed on top of the hydrous-equilibrated termination. No hydrogen atoms are depicted. Modified after Eng et al. (2000).

Figure 3. Top: images of calculated $3 \mathrm{~nm} \mathrm{ZnS}$ nanoparticles in vacuum (high surface strain) and with water coverage (strain-relaxed). Middle: PDF calculations for bulk sphalerite, and truncated spherical sphalerite nanoparticles compared with the experimental PDF from synthetic $\mathrm{ZnS}$ nanoparticles of $3 \mathrm{~nm}$ diameter. Unlike figure 1, the truncated model does not fit the damped pair correlations, due to the presence of additional disorder. Bottom: Fit to the 
experimental PDF using a model incorporating both thermal and static disorder (MSRD=mean square relative displacement; $\mathrm{SRO}=$ short range order parameter). The thermal disorder represents random variations of a given atom from its nominal lattice position, while the static disorder indicates a systematic shift in atom positions with respect to the lattice as a function of atom-atom separation. Modified from Gilbert et al. (2004).

Figure 4. Synthetic nanogoethite crystallites showing quasi-periodic image contrast features suggestive of assembly from much smaller nanoparticles. Well defined faceted nanoparticles are observed above 6-8 $\mathrm{nm}$ in size, consistent with the smallest uniform contrast regions in these crystals. Figure taken by Christopher Kim, Chapman University.

\section{KEYWORDS}

Nanoparticle, Pair distribution function, $\mathrm{ZnS}, \mathrm{TiO}_{2}$, Surface, oriented aggregation, strain 


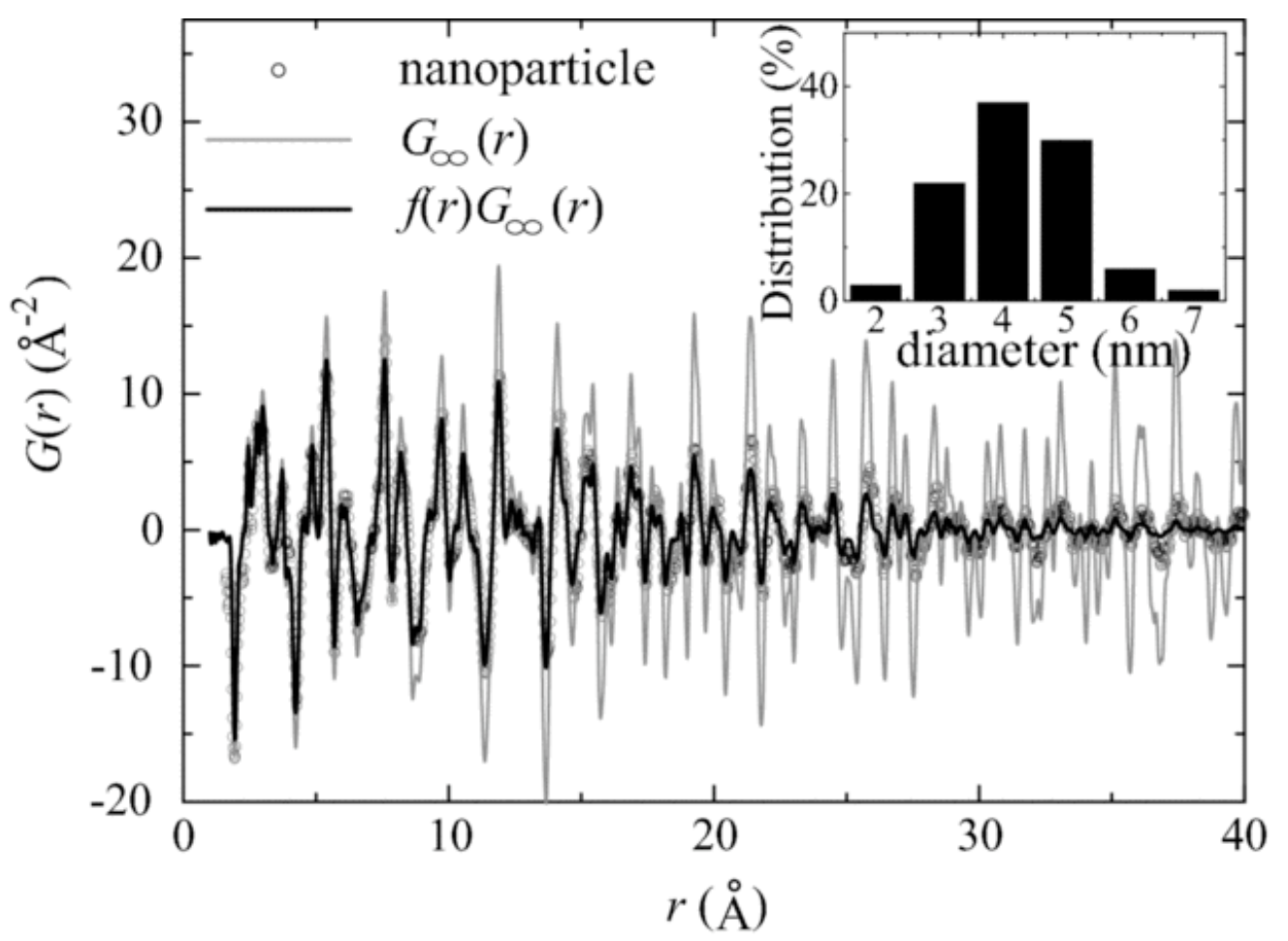

Figure 1 

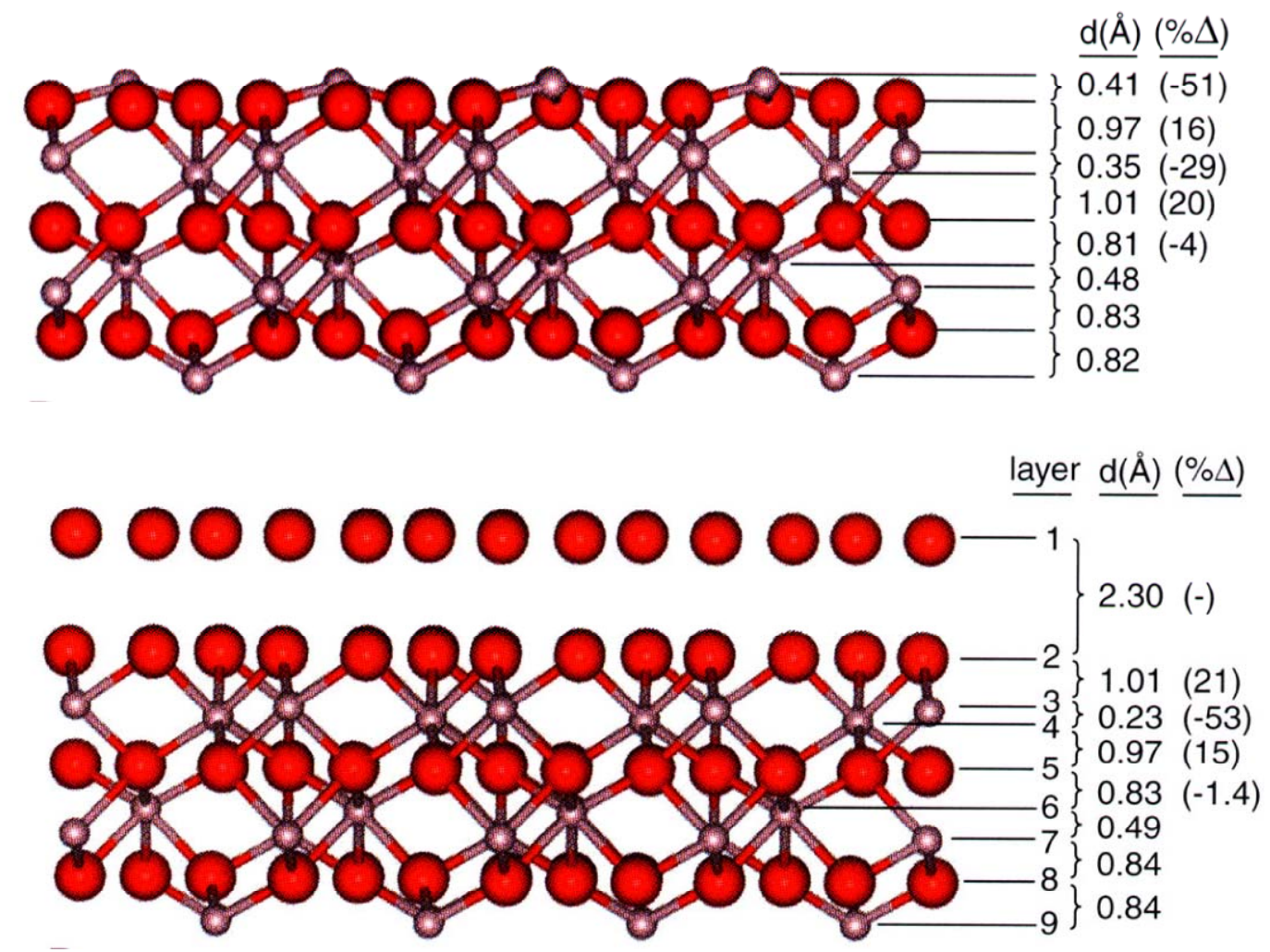

Figure 2. 

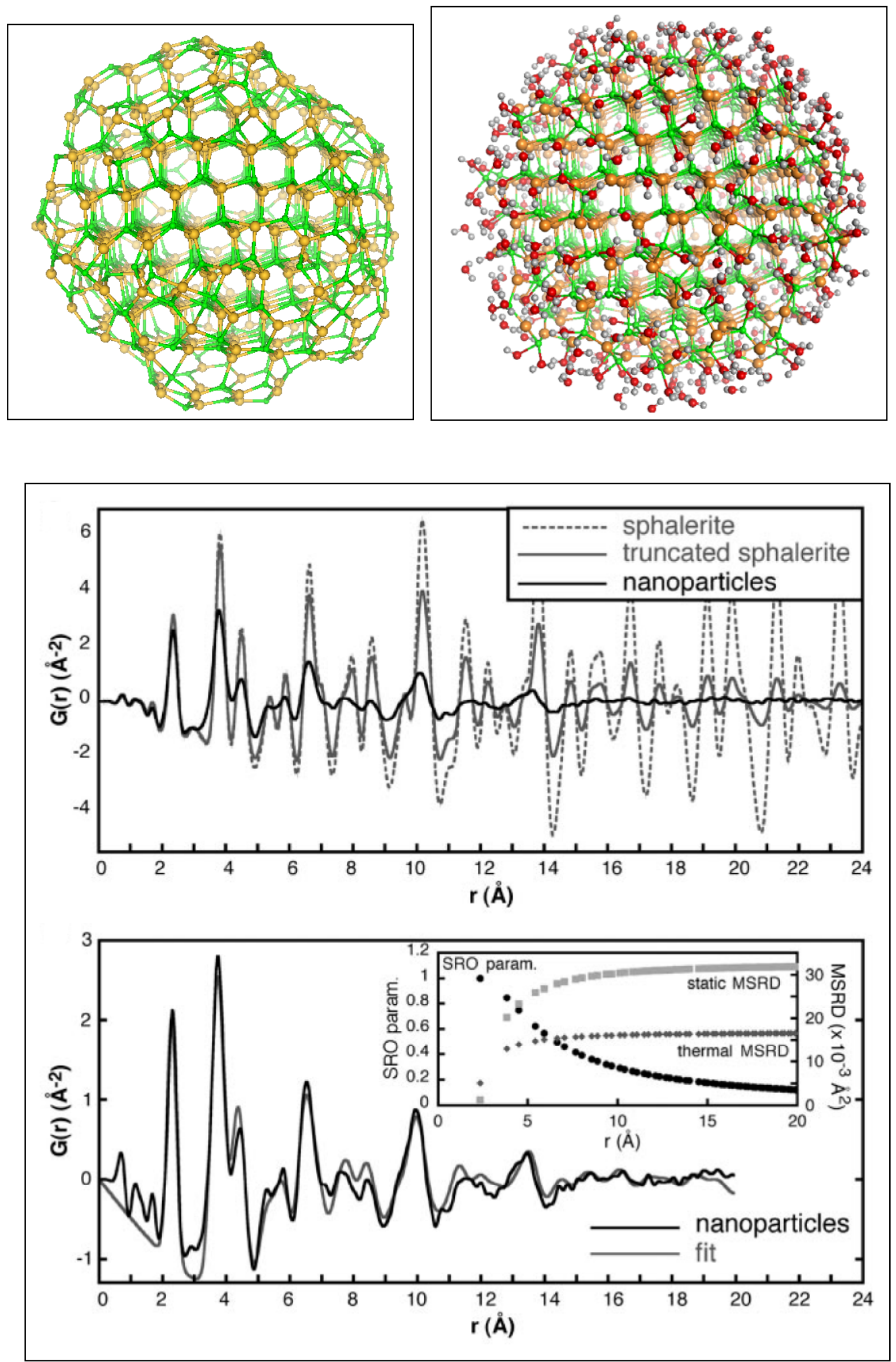

Figure 3 
Figure 4.

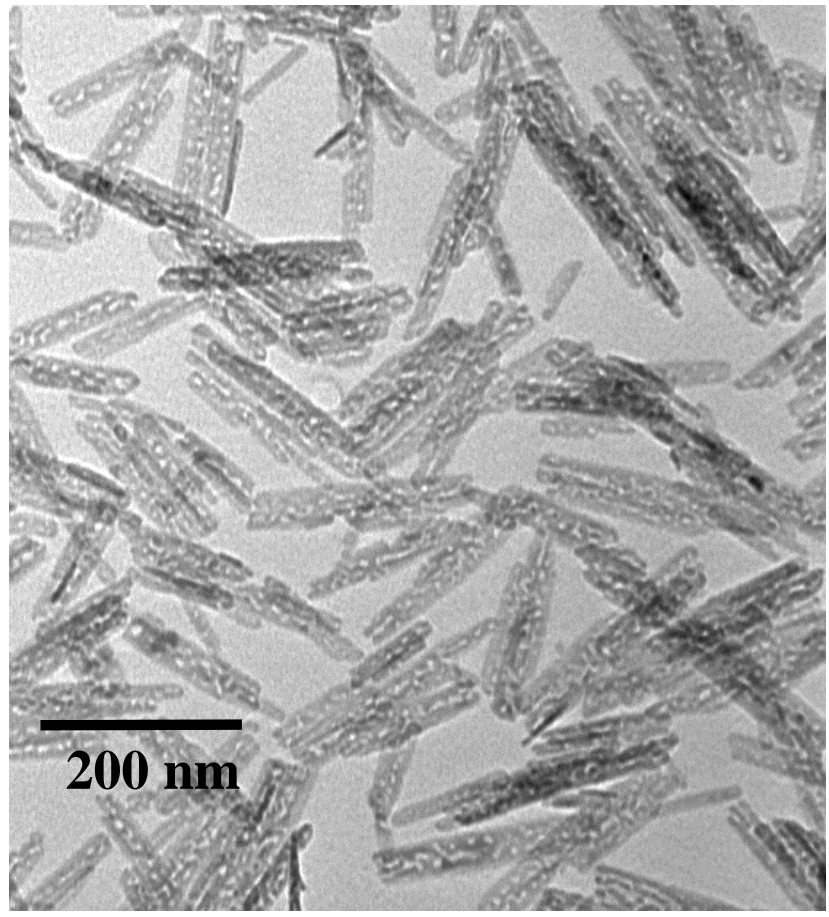

